\title{
Influence of the Type of Circulatory/Ventricular Assistance in the Primary Graft Failure and Heart Transplantation Mortality
}

\author{
Raquel López-Vilella1*, Ignacio J. Sánchez-Lázaro1, María Paz Fuset Cabanes², \\ Azucena Pajares Moncho3 , Lucía Doñate Bertolín', Ricardo Gimeno Costa², \\ Ignacio Moreno Puigdollers' ${ }^{3}$, Luis Martínez Dolz ${ }^{1}$, Iratxe Zarragoikoetxea Jauregui ${ }^{3}$, \\ Salvador Torregrosa Puerta ${ }^{4}$, Luis Almenar Bonet ${ }^{1}$
}

\footnotetext{
${ }^{1}$ Heart Failure and Transplant Unit, Hospital Universitario y Politécnico La Fe, Valencia, Spain

${ }^{2}$ Intensive Care Department, Hospital Universitario y Politécnico La Fe, Valencia, Spain

${ }^{3}$ Anesthesiology Department, Hospital Universitario y Politécnico La Fe, Valencia, Spain

${ }^{4}$ Cardiovascular Surgery Department, Hospital Universitario y Politécnico La Fe, Valencia, Spain

Email: *lopez_raqvil@gva.es
}

How to cite this paper: López-Vilella, R., Sánchez-Lázaro, I.J., Cabanes, M.P.F., Moncho, A.P., Bertolín, L.D., Costa, R.G., Puigdollers, I.M., Dolz, L.M., Jauregui, I.Z., Puerta, S.T. and Bonet, L.A. (2019) Influence of the Type of Circulatory/Ventricular Assistance in the Primary Graft Failure and Heart Transplantation Mortality. World Journal of Cardiovascular Diseases, 9, 545-552. https://doi.org/10.4236/wjcd.2019.98047

Received: June 19, 2019

Accepted: August 16, 2019

Published: August 19, 2019

Copyright $\odot 2019$ by author(s) and Scientific Research Publishing Inc. This work is licensed under the Creative Commons Attribution International License (CC BY 4.0)

http://creativecommons.org/licenses/by/4.0/

\section{c) (i) Open Access}

\begin{abstract}
OBJECTIVES: In recent years, the percentage of heart transplantation (HT) with short/medium-term assistance devices has increased. This study aims at analyzing primary graft failure and in-hospital mortality according to the type of care. MATERIAL AND METHODS: From January 2013 to December 2017 all patients undergoing urgent HT with circulatory/ventricular assistance were retrospectively and consecutively recruited. Combined transplants, retransplantations and pediatric transplants were excluded. The sample was divided in 10 groups according to the type of shot/medium term assistance devices. RESULTS: A total of 53 patients were recruited, $79 \%$ men, average age $49 \pm 13$ years. 26 patients $(51 \%)$ had mechanical ventilation at the time of the HT. Primary graft failure occurred in 20 patients $(38.5 \%)$, whilst it was more frequent in patients assisted with ECMO (8 patients in group $1(45 \%)$ and 7 in group 2 (54\%), p 0.5). 14 deaths (27\%) were registered, whereas a higher mortality was observed in the group assisted with ECMO (6 patients in group $1(34 \%)$ and 4 in group $2(31 \%), p$ 0.6). Mortality was $17 \%$ in the group of non-urgent Levitronix. CONCLUSIONS: Despite not showing statistical significance due to the low number of patients in some subgroups, hospital mortality was high in HT patients with circulatory assistance. A trend towards a higher incidence of primary graft failure and mortality in patients assisted with ECMO was observed. The direct implantation of a centrifugal pump as a
\end{abstract}


bridge to urgent transplantation could identify a subgroup with a better prognosis.

\section{Keywords}

Urgent Heart Transplantation, Short-Medium Term Circulatory/Ventricular Assistance, Primary Graft Failure

\section{Introduction}

In recent years, the percentage of urgent heart transplantation (HT) with short/medium-term assistance devices has increased. In Spain, in 2016 the urgent transplant exceeded $50 \%$ of the procedures, confirming, in that same year, the extension of the use of pretransplant ventricular assist devices that had been detected since 2009 [1]. It is complicated to compare this data with other European registries, since in most countries the use of short-medium duration mechanical assistance as a direct bridge to HT is not very common [2]. In our environment, however, the use of this type of assistance as a bridge to HT has allowed us to rescue a percentage of patients from a population that has a very high mortality rate. Nevertheless, urgent transplantation with short-term assistance devices has an impact on survival, differently depending on the type of assistance used. For example, while the survival of transplants performed with an intra-aortic balloon pump or long-term ventricular assist devices is similar with respect to elective transplantation, transplants performed with venoarterial extracorporeal membrane oxygenation (VA-ECMO) show a significantly lower survival rate to those made without any device [1]. The aim of this study is to analyze the influence of the short-medium duration circulatory/ventricular assistance type used and the implantation strategy performed on primary graft failure (PGF) and mortality in urgent HT.

\section{Material and Methods}

From January, 1st 2013 to December, 31st 2017, all patients undergoing urgent HT with circulatory/ventricular assistance were retrospectively and consecutively recruited (ECMO and LevitronixCentrimag ${ }^{\circledR}$ ). Combined transplants, retransplantations and pediatric transplants (less than 16 years old) were excluded. The total number of patients was 53. The sample was divided in 10 groups according to the type of short/medium term assistance devices used previously and at the time of transplant and taking into account the INTERMACS profile [3].

- Group 1: Patients with VA-ECMO and mechanical ventilation.

- Group 2: Patients with VA-ECMO without mechanical ventilation.

- Group 3: ECMO and, afterwards, LevitronixCentrimag ${ }^{\circledR}$, HT with both.

- Group 4: ECMO and, afterwards, LevitronixCentrimag ${ }^{\circledR}$, HT with LevitronixCentrimag ${ }^{\circledR}$. 
- Group 5: Non-urgent left LevitronixCentrimag ${ }^{\circledR}$ (patients at the INTERMACS 3).

- Group 6: Non-urgent right LevitronixCentrimag ${ }^{\circledR}$ (patients at the INTERMACS 3).

- Group 7: Non-urgent biventricular LevitronixCentrimag ${ }^{\circledR}$ (patients at the INTERMACS 3).

- Group 8: Patients who were implanted LevitronixCentrimag ${ }^{\circledR}$ and VA-ECMO (right ventricular failure), HT with both.

- Group 9: Patients who were implanted LevitronixCentrimag ${ }^{\circledR}$ and VA-ECMO (right ventricular failure), HT with LevitronixCentrimag ${ }^{\circledR}$.

- Group 10: Urgent left LevitronixCentrimag ${ }^{\circledR}$ (patients at the INTERMACS 2).

In 2014, a consensus statement released by ISHLT established diagnostic criteria for PGD [4]. The consensus classified graft dysfunction as primary graft dysfunction (PGD) or secondary graft dysfunction which had a discernible cause such as hyper-acute rejection, pulmonary hypertension, or surgical complications. PGD must be diagnosed within 24 hours of completion of surgery. Intrahospital mortality was considered as death, due to any cause, produced before hospital discharge after HT.

\section{Results}

A total of 53 patients were recruited, $79 \%$ men, average age $49 \pm 13$ years. Basal characteristic of the selected patients are shown in Table 1 and Table 2. Primary graft failure occurred in 20 patients (38.5\%), whilst it was more frequent in patients assisted with ECMO (8 patients in group 1 (45\%) and 7 in group 2 (54\%),

Table 1. Basal characteristic (I).

\begin{tabular}{|c|c|c|c|c|c|}
\hline Group & Age (years) & $\begin{array}{c}\text { Gender } \\
(\text { males }(n, \%))\end{array}$ & $\begin{array}{c}\text { Ischemic } \\
\text { cardiomyopathy }\end{array}$ & $\begin{array}{c}\text { Dilated } \\
\text { cardiomyopathy }\end{array}$ & $\begin{array}{l}\text { Valvular } \\
\text { disease }\end{array}$ \\
\hline $1(\mathrm{n}=18)$ & $49 \pm 14$ & $12(67 \%)$ & $7(39 \%)$ & $8(44 \%)$ & $2(11 \%)$ \\
\hline $2(\mathrm{n}=14)$ & $53 \pm 11$ & $11(84.6 \%)$ & $8(57 \%)$ & $3(21 \%)$ & $1(7 \%)$ \\
\hline $3(\mathrm{n}=1)$ & 16 & $1(100 \%)$ & 0 & $1(100 \%)$ & 0 \\
\hline $4(n=2)$ & $50 \pm 1$ & $1(5 \%)$ & $1(50 \%)$ & 0 & 0 \\
\hline $5(\mathrm{n}=7)$ & $49 \pm 11$ & $6(86 \%)$ & $2(29 \%)$ & $3(43 \%)$ & 0 \\
\hline $6(n=1)$ & 38 & $1(100 \%)$ & 0 & 0 & $1(100 \%)$ \\
\hline $7(\mathrm{n}=1)$ & 64 & $1(100 \%)$ & 0 & $1(100 \%)$ & 0 \\
\hline $8(\mathrm{n}=1)$ & 45 & 0 & \multicolumn{3}{|c|}{$\mathrm{n}=1(100 \%)$ hypertrophic cardiomyopathy } \\
\hline $9(\mathrm{n}=3)$ & $39 \pm 19$ & $3(100 \%)$ & 0 & $3(100 \%)$ & 0 \\
\hline $10(n=5)$ & $49 \pm 19$ & $5(100 \%)$ & $3(60 \%)$ & $2(40 \%)$ & 0 \\
\hline
\end{tabular}

Groups: 1) Patients with VA-ECMO and mechanical ventilation; 2) Patients with VA-ECMO without mechanical ventilation; 3) Group 3: ECMO and, afterwards, LevitronixCentrimag ${ }^{\circledR}$, HT with both; 4) ECMO and, afterwards, LevitronixCentrimag ${ }^{\circledR}$, HT with LevitronixCentrimag ${ }^{\circledR}$; 5) Group 5: Non-urgent left LevitronixCentrimag $^{\circledR}$ (patients at the INTERMACS 3); 6) Non-urgent right LevitronixCentrimag ${ }^{(}$(patients at the INTERMACS 3); 7) Non-urgent biventricular LevitronixCentrimag ${ }^{\circledR}$ (patients at the INTERMACS 3); 8) Patients who were implanted LevitronixCentrimag ${ }^{\circledR}$ and VA-ECMO (right ventricular failure), HT with both; 9) Patients who were implanted LevitronixCentrimag ${ }^{\circledR}$ and VA-ECMO (right ventricular failure), HT with LevitronixCentrimag ${ }^{\circledR} ; 10$ ) Urgent left LevitronixCentrimag ${ }^{\circledR}$ (patients at the INTERMACS 2). 
p 0.5). 14 deaths (27\%) were registered, whereas a higher mortality was observed in the group assisted with ECMO (6 patients in group 1 (34\%) and 4 in group 2 (31\%), p 0.6$)$. Mortality was $17 \%$ in the group of non-urgent LevitronixCentrimag $^{\circledR}$ (Table 3 and Figure 1).

Table 2. Basal characteristic (II).

\begin{tabular}{ccccccc}
\hline Group & $\begin{array}{c}\text { Age } \\
\text { (years) }\end{array}$ & $\begin{array}{c}\text { Gender } \\
\text { (males (n, \%)) }\end{array}$ & $\begin{array}{c}\text { Chronic } \\
\text { kidney disease }\end{array}$ & $\begin{array}{c}\text { Treatment with } \\
\text { inotropic agents }\end{array}$ & $\begin{array}{c}\text { Mechanic } \\
\text { ventilation }\end{array}$ & $\begin{array}{c}\text { Previous } \\
\text { sternotomy }\end{array}$ \\
\hline $1(\mathrm{n}=18)$ & $49 \pm 14$ & $12(67 \%)$ & $2(11 \%)$ & $16(89 \%)$ & $18(100 \%)$ & $1(6 \%)$ \\
$2(\mathrm{n}=14)$ & $53 \pm 11$ & $11(79 \%)$ & $1(7 \%)$ & $13(93 \%)$ & $1(7 \%)$ & $2(14 \%)$ \\
$3(\mathrm{n}=1)$ & 16 & $1(100 \%)$ & 0 & $1(100 \%)$ & 0 & 0 \\
$4(\mathrm{n}=2)$ & $50 \pm 1$ & $1(5 \%)$ & 0 & $2(100 \%)$ & $1(50 \%)$ & 0 \\
$5(\mathrm{n}=7)$ & $49 \pm 11$ & $6(86 \%)$ & $3(50 \%)$ & $5(83 \%)$ & 0 & 0 \\
$6(\mathrm{n}=1)$ & 38 & $1(100 \%)$ & $1(100 \%)$ & $1(100 \%)$ & 0 & $1(100 \%)$ \\
$7(\mathrm{n}=1)$ & 64 & $1(100 \%)$ & $1(100 \%)$ & 0 & $1(100 \%)$ & 0 \\
$8(\mathrm{n}=1)$ & 45 & 0 & 0 & $1(100 \%)$ & $1(100 \%)$ & 0 \\
$9(\mathrm{n}=3)$ & $39 \pm 19$ & $3(100 \%)$ & 0 & $3(100 \%)$ & 0 & $1(33 \%)$ \\
$10(\mathrm{n}=5)$ & $49 \pm 19$ & $5(100 \%)$ & 0 & $2(40 \%)$ & $2(40 \%)$ & $1(20 \%)$ \\
\hline
\end{tabular}

Groups: 1) Patients with VA-ECMO and mechanical ventilation; 2) Patients with VA-ECMO without mechanical ventilation; 3) Group 3: ECMO and, afterwards, LevitronixCentrimag ${ }^{\circledR}$, HT with both; 4) ECMO and, afterwards, LevitronixCentrimag ${ }^{\circledR}$, HT with LevitronixCentrimag ${ }^{\circledR}$; 5) Group 5: Non-urgent left LevitronixCentrimag $^{\circledR}$ (patients at the INTERMACS 3); 6) Non-urgent right LevitronixCentrimag ${ }^{\circledR}$ (patients at the INTERMACS 3); 7) Non-urgent biventricular LevitronixCentrimag ${ }^{\circledR}$ (patients at the INTERMACS 3); 8) Patients who were implanted LevitronixCentrimag ${ }^{\circledR}$ and VA-ECMO (right ventricular failure), HT with both; 9) Patients who were implanted LevitronixCentrimag ${ }^{\circledR}$ and VA-ECMO (right ventricular failure), HT with LevitronixCentrimag ${ }^{\circledR}$; 10) Urgent left LevitronixCentrimag ${ }^{\circledR}$ (patients at the INTERMACS 2).

Table 3. Primary graft failure and intrahospital mortality.

\begin{tabular}{cccc}
\hline Group & n (\%) & PGF (\%) & HM (\%) \\
\hline 1 & $18(34 \%)$ & $45 \%$ & $34 \%$ \\
2 & $14(26.2 \%)$ & $54 \%$ & $31 \%$ \\
3 & $1(1.9 \%)$ & 0 & 0 \\
4 & $2(3.8 \%)$ & 0 & 0 \\
5 & $7(13.2 \%)$ & $33 \%$ & $17 \%$ \\
6 & $1(1.9 \%)$ & 0 & 0 \\
7 & $1(1.9 \%)$ & 0 & 0 \\
8 & $1(1.9 \%)$ & $100 \%$ & $100 \%$ \\
9 & $3(5.7 \%)$ & 0 & 0 \\
10 & $5(9.4 \%)$ & $35 \%$ & $20 \%$ \\
\hline
\end{tabular}

Groups: 1) Patients with VA-ECMO and mechanical ventilation; 2) Patients with VA-ECMO without mechanical ventilation; 3) Group 3: ECMO and, afterwards, LevitronixCentrimag ${ }^{\circledR}$, HT with both; 4) ECMO and, afterwards, LevitronixCentrimag ${ }^{\circledR}$, HT with LevitronixCentrimag ${ }^{\circledR}$; 5) Group 5: Non-urgent left LevitronixCentrimag $^{\circledR}$ (patients at the INTERMACS 3); 6) Non-urgent right LevitronixCentrimag ${ }^{\circledR}$ (patients at the INTERMACS 3); 7) Non-urgent biventricular LevitronixCentrimag ${ }^{\circledR}$ (patients at the INTERMACS 3); 8) Patients who were implanted LevitronixCentrimag ${ }^{\circledR}$ and VA-ECMO (right ventricular failure), HT with both; 9) Patients who were implanted LevitronixCentrimag ${ }^{\circledR}$ and VA-ECMO (right ventricular failure), HT with LevitronixCentrimag ${ }^{\circledR} ; 10$ ) Urgent left LevitronixCentrimag ${ }^{\circledR}$ (patients at the INTERMACS 2). PGF: Primary graft failure; HM: Hospital mortality. 


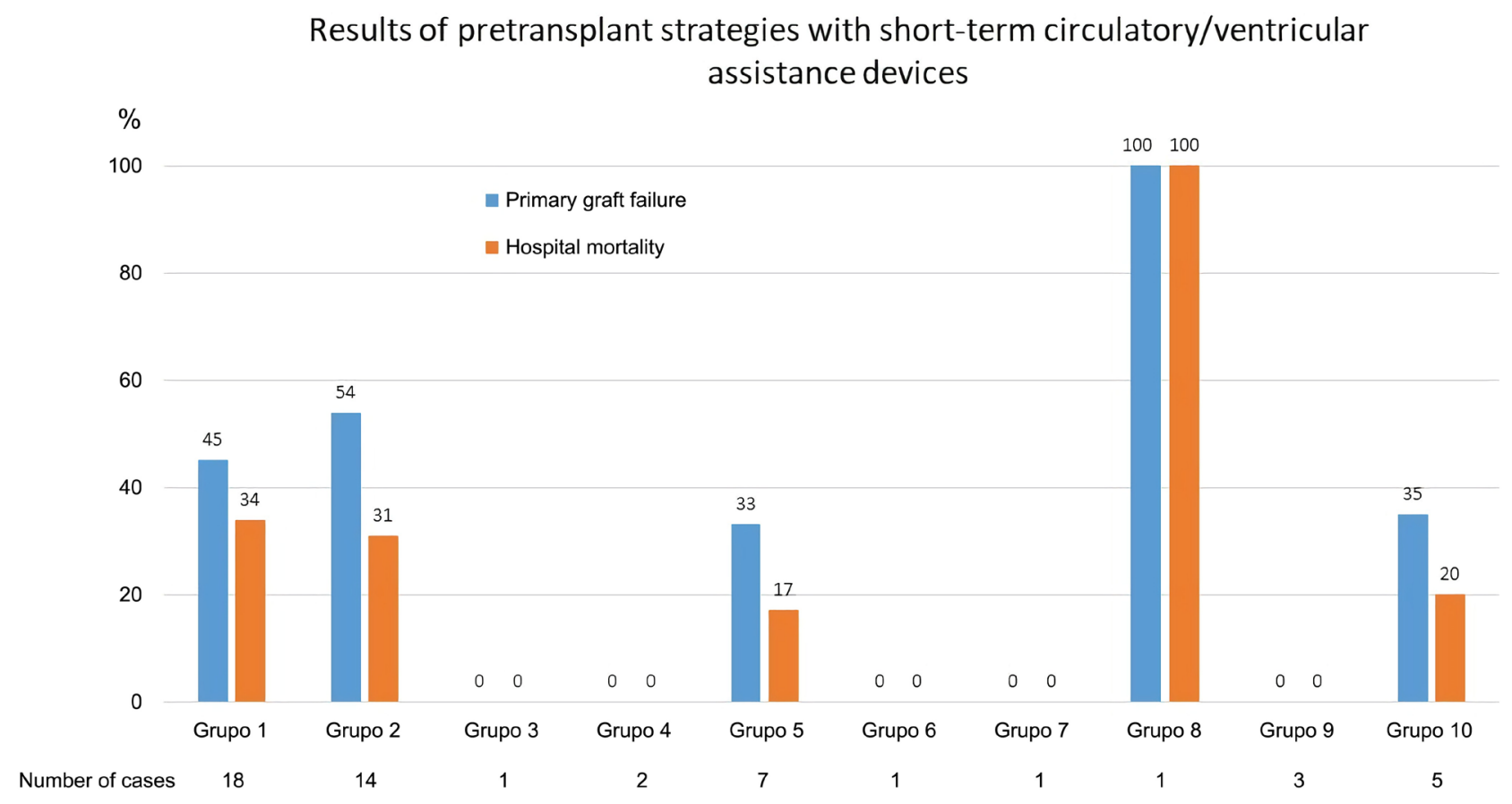

Figure 1. Groups: 1) Patients with VA-ECMO and mechanical ventilation; 2) Patients with VA-ECMO without mechanical ventilation; 3) Group 3: ECMO and, afterwards, LevitronixCentrimag ${ }^{\circledR}$, HT with both; 4) ECMO and, afterwards, LevitronixCentri$\operatorname{mag}^{\circledR}$, HT with LevitronixCentrimag ${ }^{\circledR}$; 5) Group 5: Non-urgent left LevitronixCentrimag ${ }^{\circledR}$ (patients at the INTERMACS 3); 6) Non-urgent right LevitronixCentrimag ${ }^{\circledR}$ (patients at the INTERMACS 3); 7) Non-urgent biventricular LevitronixCentrimag ${ }^{\circledR}$ (patients at the INTERMACS 3); 8) Patients who were implanted LevitronixCentrimag ${ }^{\circledR}$ and VA-ECMO (right ventricular failure), HT with both; 9) Patients who were implanted LevitronixCentrimag ${ }^{\circledR}$ and VA-ECMO (right ventricular failure), HT with LevitronixCentrimag $^{\circledR} ; 10$ ) Urgent left LevitronixCentrimag ${ }^{\circledR}$ (patients at the INTERMACS 2).

\section{Discussion}

HT remains the most effective long-term treatment for advanced heart failure refractory to optimal treatment according to guidelines. However, the shortage of donors, the long waiting times and an increasing number of unstable patients have favored the development of mechanical circulatory assistance as a bridge to decision, to recovery, to the implant of long-term mechanical assistance devices or as a bridge to the candidacy to HT. In this study, we analyzed the results of 53 patients transplanted in urgent code under support with circulatory/ventricular assistance of short-medium duration. In this sense, the most used assists in patients in INTERMACS 1 - 3 are the ECMO-VA and the LevitronixCentrimag ${ }^{\circledR}$ [5], which allow the recovery and stabilization of the patient in cardiogenic shock for days or weeks. Despite this, the mortality of these patients continues to be around $50 \%$, mainly due to shock prior to implantation and also due to the complications associated with these assistances [5] [6]. In our study, $60 \%$ of the urgent HT in the 5 years of recruitment were performed with VA-ECMO. This is a higher percentage than in other series in our setting [1], probably because VA-ECMO strategy was first initiated as a bridge to HT in our center, and in January 2016 the first LevitronixCentrimag ${ }^{\circledR}$ was implanted. In another more recent series, the percentage of patients with $\mathrm{V}$-ECMO at the time of inclusion in the emergency 
waiting list was similar, around 58\% (ASIS-TC [7]). VA-ECMO is associated with worse survival after the HT [1] [5] [6]. The results of our study coincide with these results, since the mortality of patients transplanted with VA-ECMO was higher than that of the other groups (34\% in group 1 and $31 \%$ in group 2). The longer the time of assistance with VA-ECMO, the survival is even lower [8]. Keep in mind that they are usually patients in INTERMACS 2 , which often require mechanical ventilation and high doses of inotropes. In addition, complications associated with prolonged ECMO support should be taken into account, such as insufficient left ventricular discharge, prolonged immobilization, vascular complications, coagulopathy, etc. In $22.7 \%$ of the patients, LevitronixCentrimag ${ }^{\circledR}$ was implanted in a non-urgent way (patients dependent on inotropes at the INTERMACS 3), a similar percentage to that recorded in other series of our environment [7]. Mortality was lower (17\%) in this group of patients in whom the assistance was more elective (INTERMACS 3), and it was intermediate between the two groups in those patients whom LevitronixCentrimag ${ }^{\circledR}$ were implanted with urgently (INTERMACS 2). There were no deaths between the patients with VA-ECMO and afterwards LevitronixCentrimag ${ }^{\circledR}$ (bridge to bridge), but we have to take into account that we have only selected the patients who did reach the HT. The results were good in patients with right and biventricular LevitronixCentri$\mathrm{mag}^{\circledR}$, but there was only one patient in each of these groups. Regarding PGF, it continues to be a frequent early complication of $\mathrm{HT}$ and is associated with an increase in mortality. Likewise, PGF was more frequent in patients assisted with VA-ECMO (8 patients in group $1(45 \%)$ and 7 in group $2(54 \%), p$ 0.5), in a manner consistent with similar studies [9]. PGF and death occurred also in the only patient included in group 8 (implant in the same act of LevitronixCentri$\mathrm{mag}^{\circledR}$ and ECMO-VA, HT with both assistive devices), but since it is a single patient it is a result difficult to interpret. It must be taken into account that these results are obtained from a selected population of young patients (average of 49 years), with a relatively low prevalence of comorbidities prior to the HT, and discarding those patients who died during circulatory support without reaching transplantation. Under these conditions, it seems that the direct implantation of a medium-term device (LevitronixCentrimag ${ }^{\circledR}$ ) and the consequent decrease in the use of VA-ECMO make possible a state prior to the more stable transplant and are associated with better results as a direct bridge to urgent HT.

Our study has limitations, basically it is a unicentric study, with a small sample and retrospectively analyzed. The small number of patients makes difficult to reach statistical significance, however, we see results that coincide with those of other similar series, and allow us to get an idea of the influence of the type of short or medium term assistance used as direct bridge to emergency HT, a strategy not common in the global transplantation centers. Finally, our results may not be applicable to other systems with longer wait times for urgent HT and greater availability of long-term ventricular assist devices. 


\section{Conclusion}

In conclusion, despite not showing statistical significance due to the low number of patients in some subgroups, hospital mortality was high in HT patients with circulatory assistance. A trend towards a higher incidence of primary graft failure and mortality in patients assisted with VA-ECMO was observed. The direct implantation of a centrifugal pump as a bridge to urgent transplantation could identify a subgroup with a better prognosis. Studies with a larger number of patients are necessary to define the best strategy for the management of patients in cardiogenic shock with short-medium-term mechanical assistance aimed at urgent heart transplantation.

\section{Conflicts of Interest}

The authors declare no conflicts of interest regarding the publication of this paper.

\section{References}

[1] González-Vílchez, F., Gómez-Bueno, M., Almenar-Bonet, L., et al. (2017) Spanish Heart Transplant Registry. 28th Official Report of the Spanish Society of Cardiology Working Group on Heart Failure (1984-2016). Revista Española de Cardiología, 70, 1098-1109. https://doi.org/10.1016/j.rec.2017.10.016

[2] Lund, L.H., Edwards, L.B., Dipchand, A.I., et al. (2016) The Registry of the International Society for Heart and Lung Transplantation: Thirty-Third Adult Heart Transplantation Report-2016; Focus Theme: Primary Diagnostic Indications for Transplant. The Journal of Heart and Lung Transplantation, 35, 1158-1169. https://doi.org/10.1016/j.healun.2016.08.017

[3] Stevenson, L.W., Pagani, F.D., Young, J.B., et al. (2009) INTERMACS Profiles of Advanced Heart Failure: The Current Picture. The Journal of Heart and Lung Transplantation, 28, 535-541. https://doi.org/10.1016/j.healun.2009.02.015

[4] Kobashigawa, J., Zuckermann, A., Macdonald, P., et al. (2014) Report from a Consensus Conference on Primary Graft Dysfunction after Cardiac Transplantation. The Journal of Heart and Lung Transplantation, 33, 327-340. https://doi.org/10.1016/j.healun.2014.02.027

[5] Sánchez-Enrique, C., Jorde Ulrich, P. and González-Costello, J. (2017) Trasplante cardiaco y soporte circulatorio mecánico para pacientes con insuficiencia cardiaca avanzada. Revista Española de Cardiología, 70, 371-381. https://doi.org/10.1016/j.recesp.2016.10.032

[6] Takayama, H., Takeda, K., Doshi, D., et al. (2014) Short-Term Continuous-Flow Ventricular Assist Devices. Current Opinion in Cardiology, 29, 266-274. https://doi.org/10.1097/HCO.0000000000000060

[7] Barge-Caballero, E., Almenar-Bonet, L., Gonzalez-Vilchez, F., et al. (2017) Clinical Outcomes of Temporary Mechanical Circulatory Support as Direct Bridge to Heart Transplantation: A Nationwide Spanish Registry. European Journal of Heart Failure, 20, 178-186. https://doi.org/10.1002/ejhf.956

[8] López-Vilella, R., Sánchez-Lázaro, I., FusetCabanes, M.P., et al. (2017) Análisis de la mortalidad hospitalaria y fallo agudo del injerto en el trasplante cardíaco urgente según el tipo y la duración de la asistencia circulatoria/ventricular. Revista Española 
de Cardiología, 70, 359.

[9] Avtaar Singh, S.S., Banner, N.R., Rushton, S., Simon, A.R., Berry, C. and Al-Attar, N. (2018) ISHLT Primary Graft Dysfunction Incidence, Risk Factors and Outcome: A UK National Study. Transplantation, 103, 336-343.

https://doi.org/10.1097/TP.0000000000002220 